\title{
Capteur de gaz à base de nanoparticules : synthèse de nano-objets par voie chimique, intégration et caractérisation de capteurs élaborés en salle blanche
}

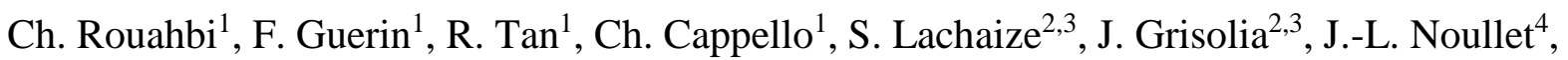 \\ J. Carrey ${ }^{2}$, Ph. Bourdeu-Daguerre ${ }^{1}$, F. Gessinn ${ }^{1}$, M. Respaud ${ }^{1,2,3}$ \\ ${ }^{1}$ AIME pôle CNFM de Toulouse, INSA de Toulouse, France \\ ${ }^{2}$ LPCNO-INSA, INSA de Toulouse, France \\ ${ }^{3}$ Département de Physique, INSA de Toulouse, France \\ Contact : tan@aime-toulouse.fr
}

\begin{abstract}
Le but de cette formation est de proposer à des étudiants (de niveau Master/Ingénieur, ou plus : doctorants, formation continue,...) une étude expérimentale complète sur les capteurs de gaz à base de nanoparticules (NPs). Ces dispositifs permettent en outre d'illustrer les intérêts de l'utilisation de NPs dans le domaine technologique.
\end{abstract}

\section{Introduction}

Le but de cette formation est de proposer à des étudiants (de niveau Master/Ingénieur, ou plus : doctorants, formation continue, etc.), une étude expérimentale complète sur les capteurs de gaz à base de nanoparticules (NPs). Ces dispositifs permettent en outre d'illustrer les intérêts de l'utilisation de NPs dans le domaine technologique. Le fonctionnement des capteurs de gaz étant basé sur la modification des propriétés chimiques (et de conduction) de leur surface, l'intégration de NPs, qui présentent un ratio surface/volume exposé très grand, conduit alors à une sensibilité des capteurs accrue. Ce stage, à fort caractère interdisciplinaire (chimie, physique, technologie), permet en outre d'aborder différents domaines scientifiques et technologiques via la réalisation complète de tels dispositifs, et ce, dans un environnement de salle blanche (AIME de Toulouse) :

- fabrication des puces suivant des procédés conventionnels en micro-électronique (technologie du silicium),

- synthèse de NPs (par voie chimique : simplicité de mise en œuvre et faible coût),

- intégration et adressage localisés des nano-objets sur les puces, pour l'obtention des capteurs,

- caractérisations électriques des capteurs finis (sous gaz).

\section{Fabrication des puces (1 jour et demi)}

La première étape du stage concerne la réalisation des puces. Elle permet aux étudiants d'avoir une vue complète sur les différentes étapes de micro-électronique, des procédés d'élaboration (dépôt de résine, photolithographie, alignement de masque, gravure de métal/SiO2/Si par attaque chimique/physique, dopage, etc.) au montage et connexion sur boitier.

Le design global d'une puce supportant les capteurs est présentée sur la Figure 1-A/. Au total, 256 puces seront réalisées par l'étudiant sur une plaquette de silicium 2 pouces.

Les puces supportent 4 éléments :

- deux capteurs en aluminium composés de deux électrodes inter-digités, espacées de $5 \mu \mathrm{m}$ ( $c f$. Figure 1-C/). Cette géométrie en peigne présente deux avantages : i/ elle augmente la probabilité de piéger des NPs entre les électrodes et ii/ les courants mesurés sur des réseaux de 
NPs étant faibles, elle permet d'obtenir un grand nombre de chemins de conduction en parallèle, ce qui réduit la résistance totale des capteurs,

- une résistance en polysilicium localisée sous les capteurs, enfouie et isolée électriquement par une couche de $\mathrm{SiO}_{2}$. Cette résistance permet notamment de chauffer la puce (jusqu'à $250^{\circ} \mathrm{C}$ ), afin de travailler à une température optimale pour obtenir une meilleure sensibilité des capteurs. La réalisation de rampes en température permet également de "ré-initialiser » l'état chimique de surface en facilitant la désorption des molécules adsorbées lors de la phase de test en présence de gaz,

- une résistance en aluminium, qui permet de mesurer la température à la surface du capteur.
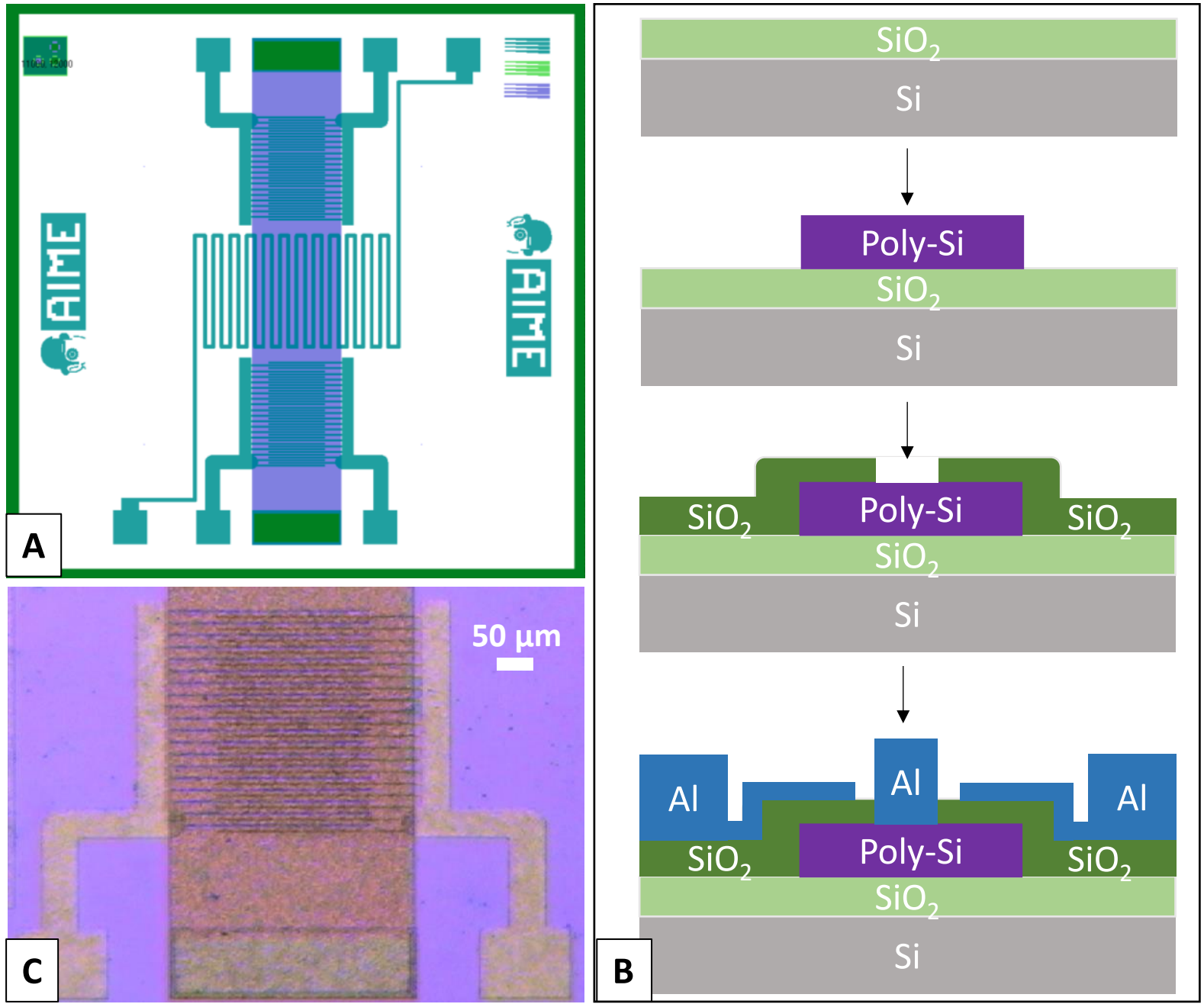

Fig.1. Elaboration du dispositif A/ design des capteurs de gaz comprenant les 3 niveaux de masquage. En bleu : les capteurs inter-digités, et la résistance en aluminium (en surface). En violet : la résistance de chauffe en polysilicium (enfouie). En vert : l'oxyde de silicium. B/ étapes de fabrication des capteurs : réalisation de la résistance chauffante de polysilicium, ouverture des contacts, puis métallisation à l'aluminium (réalisation des capteurs inter-digités et de la résistance de surface). $\mathrm{C}$ / cliché optique du capteur et de la résistance de polysilicium enfouie.

Trois étapes de masquage seront nécessaires pour la fabrication des puces chauffantes. Les principales étapes technologiques sont illustrées dans la Figure 1-B/. Brièvement, après oxydation de la plaquette de $\mathrm{Si}$, une couche de polysilicium de $300 \mathrm{~nm}$ est déposée. Le premier niveau de masquage permet alors de créer la résistance chauffante, après la première photolithographie (insolation de la résine) et la gravure du polysilicium. Le polysilicium est ensuite dopé au phosphore, afin d'obtenir une résistance de $100 \Omega$. Cette dernière est ensuite 
recouverte d'une couche de $\mathrm{SiO}_{2}$ qui sera à son tour gravée (deuxième niveau de masquage) afin d'ouvrir les contacts de la résistance. Enfin, une couche d'aluminium de $600 \mathrm{~nm}$ est déposée. La gravure de l'aluminium après le troisième masquage donne lieu aux peignes interdigités et à la résistance d'Al (sonde de température).

Synthèse des nanoparticules (1 jour):

Le but de cette étape est d'initier les étudiants à la synthèse de nano-objets par voie chimique. Certains oxydes semi-conducteurs utilisés dans les capteurs chimiques sont déjà bien connus pour leur forte réponse en présence de gaz: $\mathrm{SnO}_{2}, \mathrm{ZnO}$ mais aussi $\mathrm{WO}_{3}$. C'est ce dernier matériau sous forme de NPs que nous proposons d'étudier dans ce stage.

La synthèse des NPs de $\mathrm{WO}_{3}$ est effectuée en milieu aqueux, par une méthode de condensation classique. Cette méthode d'élaboration a été choisie car elle est relativement aisée (facile à mettre en œuvre, avec des équipements légers et de routine en chimie), mais aussi parce qu'elle présente peu de risques pour les étudiants par rapport à d'autres type de synthèse (conditions douces, à $180^{\circ} \mathrm{C}$ ). Brièvement, un précurseur de tungstène $\mathrm{Na}_{2} \mathrm{WO}_{4}-2 \mathrm{H}_{20}$ (6 grammes) est ajouté dans un mélange d'acide chlorhydrique et d'eau désionisée (DI), en présence de $\mathrm{Na}_{2} \mathrm{SO}_{4}$ en proportion de 4/5/30 molaire. La solution est ensuite transférée dans un réacteur qui sera porté à $180^{\circ} \mathrm{C}$ pendant $4 \mathrm{~h}$. Après ce délai, la solution récupérée est centrifugée et le solide obtenu est dispersé à nouveau dans de l'eau DI afin d'obtenir une solution colloïdale. Les nanoparticules obtenues dans ces conditions de synthèse ont une forme allongée avec un diamètre moyen de $\sim 100 \mathrm{~nm}$, pour une longueur comprise entre 1-2 $\mu \mathrm{m}$ ( $c f$. Figure 2-C/et 2$\mathrm{D} /)$.

\section{Intégration des nano-objets dans les capteurs (1/2 journée)}

Les puces fabriquées lors de la première étape sont découpées à l'aide d'une scie diamantée. Les étudiants choisissent alors une puce, après un contrôle optique, qui sera montée puis connectée (par bonding) sur un boitier de type TO5 (Figure 2-A/). L'étape qui suit permettra aux étudiants de prendre conscience de la difficulté majeure qui se pose alors, à savoir la manipulation et l'intégration d'objets de taille nanométrique dans des dispositifs de mesure.

Plusieurs méthodes sont recensées dans la littérature : le simple dépôt par goutte, qui reste aléatoire et présente donc peu de chances de créer des chemins de conduction électrique entre les électrodes, et le dépôt convectif, donnant lieu à de meilleurs taux de couverture des dépôts de NPs, mais qui nécessiterait des étapes supplémentaires (préparation spécifiques des substrats, nettoyage des électrodes pour le bonding après dépôt, etc.). Dans les deux cas, aucune de ces techniques ne permet réellement un vrai adressage de NPs dans une zone d'intérêt localisée, à savoir dans notre cas, entre les peignes inter-digitées. Nous avons donc opté pour la méthode de diélectrophorèse. Cette technique consiste à attirer les NPs via l'application d'un champ électrique alternatif aux bornes des électrodes de mesure. Elle permet donc un piégeage électrique mieux localisé entre les peignes.

Un montage expérimental a donc été spécialement conçu pour cette étape (Figure 2-B/). Un GBF porté à $20 \mathrm{VCC}$ à une fréquence de $100 \mathrm{kHz}$ est branché aux bornes des capteurs. L'observation en temps réel d'amas de NPs attirés par diélectrophorèse est réalisable à l'aide d'une optique focalisée sur les électrodes. Les capteurs obtenus après dépôt sont présentés dans la Figure 2-C/ et D/. Nous pouvons remarquer que cette méthode est très efficace, avec un taux de couverture entre les électrodes proches de $100 \%$, et très peu de NPs déposés hors de la zone des électrodes. 

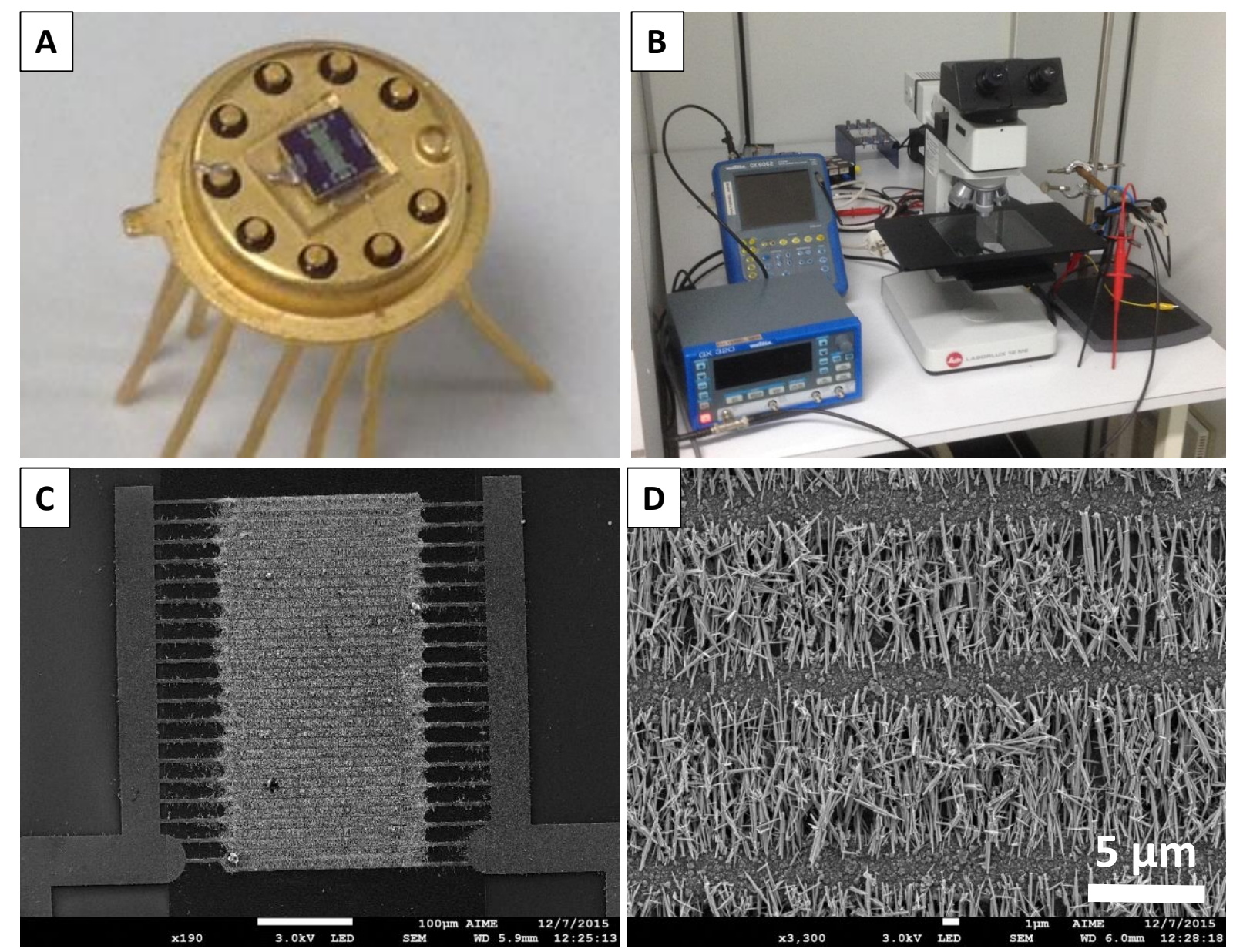

Fig.2. Intégration des nano-objets A/ montage des puces sur boitier TO5. B/ Banc de dépôt des nanoparticules par diéléctrophorèse. Microscopie électronique (SEM-FEG) des capteurs après intégration des nanofils de $\mathrm{WO}_{3}$ $\mathrm{C} /$, et à fort grandissement $\mathrm{D} /$.

\section{$\underline{\text { Caractérisation des capteurs ( } 1 / 2 \text { journée) }}$}

La dernière étape consiste à caractériser électriquement les capteurs finis. Un banc expérimental spécialement dédié pour des mesures de résistance sous balayage de gaz a été développé (Figure 3-A/). Des lignes d'air sec, d'ammoniac et d'éthanol sont reliées à une enceinte étanche dans laquelle sont connectés les capteurs. Le balayage des gaz est piloté à l'aide d'électrovannes et les mesures de résistance via l'interfaçage de multimètres (Keithley 2400).

Le protocole de mesure est le suivant : les capteurs sont d'abord portés puis maintenus durant toute la manipulation à $250^{\circ} \mathrm{C}$, via le passage d'un courant à travers la résistance de polysilicium ( 120 mA). Cette chauffe permet dans un premier temps de désorber des molécules à surface des NPs, puis dans un second temps de faciliter le « reset » de la surface après exposition à un gaz d'intérêt. Après stabilisation de la température à $250^{\circ} \mathrm{C}$, la résistance des capteurs présentent un plateau (typiquement $\sim 50 \mathrm{M} \Omega$ pour une tension appliquée de $20 \mathrm{~V}$ ). Cette valeur servira de seuil à atteindre avant l'injection d'un gaz (reset du capteur).

La figure 3-B/ présente la variation de résistance des capteurs de gaz à base de NPs de $\mathrm{WO}_{3}$ sous l'injection d'ammoniac (100 ppm) et d'éthanol (1000 ppm). Les variations de résistance atteignent un facteur 2. 


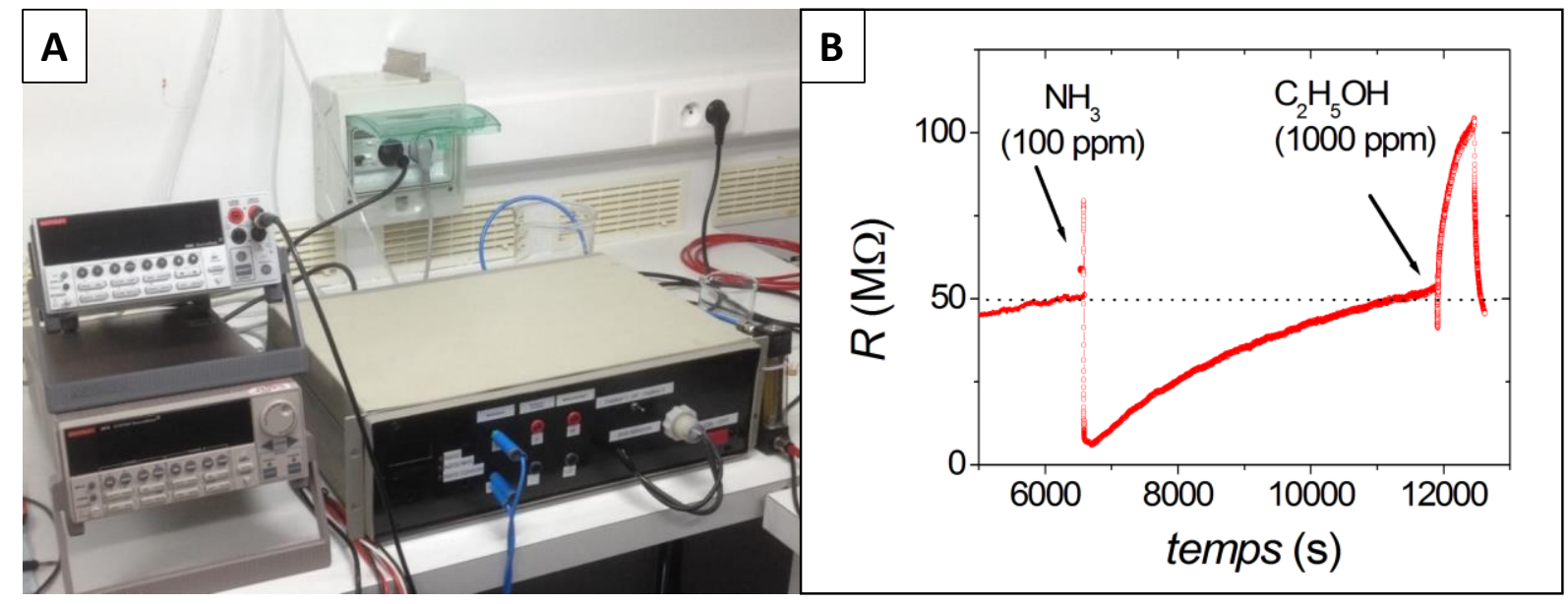

Fig.3. caractérisation des capteurs A/ banc de mesure électrique des capteurs, intégrant une enceinte permettant le balayage de différents gaz (air sec, ammoniac et éthanol). B/ Variation de la résistance des capteurs de WO3 en fonction du temps, sous la présence d'ammoniac et d'éthanol.

D'autres gaz pourront être injectés par la suite (fort caractère oxydant ou réducteur), notamment afin de mieux déterminer la sélectivité des capteurs. De même, le procédé d'intégration de NPs que nous avons mis en place nous permettra de comparer la réponse et la sensibilité de différents matériaux.

\section{Conclusion}

En conclusion, nous avons développé une formation pluridisciplinaire permettant de s'adresser à un public très large. En effet, en plus de couvrir les techniques conventionnelles en microélectronique, ce stage permet aux étudiants de réaliser un dispositif intégrant des nanoparticules synthétisées par voie chimique, avec, au final, une application concrète dans le domaine de la nanotechnologie.

\section{Remerciements}

La création de ce TP innovant a reçu le soutien du GIP-CNFM et du projet IDEFI-FINMINA ANR 2011-IDFI-0017 dans le cadre du programme Grands Investissements d'Avenir (PIA1). 